\title{
q-Analog of shock soliton solution
}

\author{
Sengul Nalci and Oktay K Pashaev \\ Department of Mathematics, Izmir Institute of Technology, Urla Izmir, 35430, Turkey \\ E-mail: oktaypashaev@iyte.edu.tr
}

Received 25 June 2010, in final form 1 September 2010

Published 11 October 2010

Online at stacks.iop.org/JPhysA/43/445205

\begin{abstract}
Based on Jackson's q-exponential function, we introduce a q-analog of Hermite and Kampe de Feriet polynomials. It allows us to introduce and solve the q-heat equation in terms of q-Kampe de Feriet polynomials with arbitrary number of moving zeros, and to find an operator solution for the initial value problem. By the q-analog of Cole-Hopf transformation we find a new q-Burgers-type nonlinear heat equation with cubic nonlinearity, such that in the $q \rightarrow 1$ limit it reduces to the standard Burgers equation. We construct exact solutions for the q-Burgers equation in the form of moving poles, singular and regular q-shock soliton solutions. A novel, self-similarity property of the stationary q-shock soliton solution is found.
\end{abstract}

PACS numbers: $02.30 . \mathrm{Gp}, 02.30 . \mathrm{Ik}, 05.45 . \mathrm{Yv}$

(Some figures in this article are in colour only in the electronic version)

\section{Introduction}

It is well known that the Burgers' equation in one dimension can be reduced via the Cole-Hopf transformation to the linear heat equation. It allows one to solve the initial value problem for the Burgers equation and get exact solutions in the form of shock solitons and their scattering. In this paper we introduce the q-difference Burgers-type equation with cubic nonlinearity, linearizable in terms of the q-heat equation. Based on the Jackson q-exponential function, we introduce q-analogs of the Hermite and the Kampe de Feriet polynomials, representing moving poles solution for the q-Burgers equation. Then we derive the operator solution of the initial value problem (IVP) for the q-Burgers equation in terms of the IVP for the q-heat equation. We construct several particular solutions of our q-Burgers-type equation in the form of singular and regular q-shock solitons. It turns out that the static q-shock soliton solution of our equation shows remarkable self-similarity property in the space coordinate $x$.

\section{2. q-exponential function}

The q-number $[n]_{q}$ corresponding to the ordinary number $n$ is defined by [1]

$$
[n]_{q}=\frac{q^{n}-1}{q-1},
$$


where $q$ is a parameter, so that $n$ is the limit of $[n]_{q}$ as $q \rightarrow 1$. A few examples of q-numbers are given here: $[0]_{q}=0,[1]_{q}=1,[2]_{q}=1+q,[3]_{q}=1+q+q^{2}$. In terms of these q-numbers, the Jackson q-exponential function $e_{q}(x)$ [3] (see [2], p 126) is defined by

$$
e_{q}(x)=\sum_{n=0}^{\infty} \frac{x^{n}}{[n]_{q} !},
$$

where $[n]_{q} !=[1]_{q}[2]_{q} \cdots[n]_{q}$. For $q>1$ it is an entire function of $x$ and when $q \rightarrow 1$ it reduces to the standard exponential function $e^{x}$. The q-exponential function can also be expressed in terms of the infinite product

$$
e_{q}(x)=\prod_{n=0}^{\infty} \frac{1}{\left(1-(1-q) q^{n} x\right)}=\frac{1}{(1-(1-q) x)_{q}^{\infty}},
$$

when $q<1$ and

$$
e_{q}(x)=\prod_{n=0}^{\infty}\left(1+\left(1-\frac{1}{q}\right) \frac{1}{q^{n}} x\right)=\left(1+\left(1-\frac{1}{q}\right) x\right)_{1 / q}^{\infty}
$$

when $q>1$. Thus, the q-exponential function for $q<1$ has the infinite set of simple poles

$$
x_{n}=\frac{1}{q^{n}(1-q)}, \quad n=0,1, \ldots
$$

and for $q>1$ the infinite set of simple zeros

$$
x_{n}=-\frac{q^{n+1}}{(q-1)}, \quad n=0,1, \ldots
$$

The q-derivative is defined as

$$
D_{x} f(x)=\frac{f(q x)-f(x)}{(q-1) x},
$$

and when $q \rightarrow 1$ it reduces to the standard derivative $D_{x} f(x) \rightarrow f^{\prime}(x)$. Using the definition of $q$-derivative one can easily see that

$$
\begin{aligned}
& D_{x}\left(a x^{n}\right)=a[n]_{q} x^{n-1}, \\
& D_{x} e_{q}(a x)=a e_{q}(a x) .
\end{aligned}
$$

\section{3. q-Hermite polynomials}

We define the q-analog of Hermite polynomials by the generating function

$$
e_{q}\left(-t^{2}\right) e_{q}\left([2]_{q} t x\right)=\sum_{n=0}^{\infty} H_{n}(x ; q) \frac{t^{n}}{[n]_{q} !} .
$$

From the defining identity (10) it is not difficult to derive for the q-Hermite polynomials an explicit sum formula

$$
H_{n}(x ; q)=\sum_{k=0}^{[n / 2]} \frac{(-1)^{k}[n]_{q} !}{[k]_{q} ![n-2 k]_{q} !}\left([2]_{q} x\right)^{n-2 k} .
$$

This explicit sum makes it transparent in which way our polynomials $H_{n}(x ; q) q$-extend the $H_{n}(x)$ and how they are different from the known ones in the literature. By q-differentiating the 
generating function (10) with respect to $x$ and $t$ we derive two-term and three-term recurrence relations correspondingly

$D_{x} H_{n}(x ; q)=[2]_{q}[n]_{q} H_{n-1}(x ; q)$,
$H_{n+1}(x ; q)=[2]_{q} x H_{n}(x ; q)-[n]_{q} H_{n-1}(q x ; q)-[n]_{q} q^{\frac{n+1}{2}} H_{n-1}(\sqrt{q} x ; q)$.

From this generating function we have the special values

$$
\begin{aligned}
& H_{2 n}(0 ; q)=(-1)^{n} \frac{[2 n]_{q} !}{[n]_{q} !}, \\
& H_{2 n+1}(0 ; q)=0
\end{aligned}
$$

and the parity relation

$$
H_{n}(-x ; q)=(-1)^{n} H_{n}(x ; q) .
$$

To write the three-term recurrence relation in the local form for the same argument $x$, we use delation operator

$$
M_{q}=q^{x \frac{\mathrm{d}}{\mathrm{d} x}}
$$

so that

$$
M_{q} f(x)=f(q x)
$$

and relation (13) can be rewritten as

$$
H_{n+1}(x ; q)=[2]_{q} x H_{n}(x ; q)-[n]_{q}\left(M_{q}+q^{\frac{n+1}{2}} M_{\sqrt{q}}\right) H_{n-1}(x ; q) .
$$

Substituting (12) into (19) we get

$$
H_{n+1}(x ; q)=\left([2]_{q} x-\frac{M_{q}+q^{\frac{n+1}{2}} M_{\sqrt{q}}}{[2]_{q}} D_{x}\right) H_{n}(x ; q) .
$$

By the recursion, starting from $n=0$ and $H_{0}(x)=1$ we have the next representation for the q-Hermite polynomials

$$
H_{n}(x ; q)=\prod_{k=1}^{n}\left([2]_{q} x-\frac{M_{q}+q^{\frac{k}{2}} M_{\sqrt{q}}}{[2]_{q}} D_{x}\right) \cdot 1 .
$$

We note that the generating function and the form of our q-Hermite polynomials are different from the known ones in the literature [2, 4-6]. Moreover, the three-term recurrence relation (13) is q-nonlocal and different from the known ones for orthogonal polynomial sets [7].

In the above expression the operator

$$
M_{q}+q^{\frac{n}{2}} M_{\sqrt{q}}=2 q^{\frac{n}{4}} q^{\frac{3}{4} x \frac{\mathrm{d}}{\mathrm{d} x}} \cosh \left[\left(\ln q^{\frac{1}{4}}\right)\left(x \frac{\mathrm{d}}{\mathrm{d} x}-n\right)\right]
$$

is expressible in terms of the q-spherical means as

$$
\cosh \left[(\ln q) x \frac{\mathrm{d}}{\mathrm{d} x}\right] f(x)=\frac{1}{2}\left(f(q x)+f\left(\frac{1}{q} x\right)\right) .
$$

By notation for the $q$-shifted product [1]

$$
(x-a)_{q}^{n}=(x-a)(x-q a) \cdots\left(x-q^{n-1} a\right), \quad n=1,2, \ldots
$$

which we now apply to the noncommutative operators, so that we should distinguish the direction of multiplication, we have two cases

$$
(x-a)_{q<}^{n}=(x-a)(x-q a) \cdots\left(x-q^{n-1} a\right)
$$


and

$$
(x-a)_{q>}^{n}=\left(x-q^{n-1} a\right) \cdots(x-q a)(x-a) .
$$

Then, we can rewrite (21) shortly as

$$
H_{n}(x ; q)=\left(\left([2]_{q} x-\frac{M_{q} D_{x}}{[2]_{q}}\right)-q^{\frac{1}{2}} \frac{M_{\sqrt{q}} D_{x}}{[2]_{q}}\right)_{\sqrt{q}>}^{n} \cdot 1 .
$$

First few polynomials are

$$
\begin{aligned}
& H_{0}(x ; q)=1 \\
& H_{1}(x ; q)=[2]_{q} x \\
& H_{2}(x ; q)=[2]_{q}^{2} x^{2}-[2]_{q} \\
& H_{3}(x ; q)=[2]_{q}^{3} x^{3}-[2]_{q}^{2}[3]_{q} x \\
& H_{4}(x ; q)=[2]_{q}^{4} x^{4}-[2]_{q}^{2}[3]_{q}[4]_{q} x^{2}+[2]_{q}[3]_{q}[2]_{q^{2}} .
\end{aligned}
$$

When $q \rightarrow 1$ these polynomials reduce to the standard Hermite polynomials.

\section{1. q-Difference equation}

Applying $D_{x}$ to both sides of (20) and using recurrence formula (12) we get the q-difference equation for the q-Hermite polynomials

$\frac{1}{[2]_{q}} D_{x}\left(M_{q}+q^{\frac{n+1}{2}} M_{\sqrt{q}}\right) D_{x} H_{n}(x ; q)-[2]_{q} q x D_{x} H_{n}(x ; q)+[2]_{q}[n]_{q} q H_{n}(x ; q)=0$.

\subsection{Operator representation}

\section{Proposition 1.}

$$
e_{q}\left(-\frac{1}{[2]_{q}^{2}} D_{x}^{2}\right) e_{q}\left([2]_{q} x t\right)=e_{q}\left(-t^{2}\right) e_{q}\left([2]_{q} x t\right)
$$

Proof. By q-differentiating the q-exponential function with respect to $x$

$$
D_{x}^{n} e_{q}\left([2]_{q} x t\right)=([2] t)^{n} e_{q}\left([2]_{q} x t\right)
$$

and combining then to the sum

$$
\sum_{n=0}^{\infty} \frac{a^{n}}{[n]_{q} !} D_{x}^{2 n} e_{q}\left([2]_{q} x t\right)=\sum_{n=0}^{\infty} \frac{[2]_{q}^{2 n} a^{n} t^{2 n}}{[n]_{q} !} e_{q}\left([2]_{q} x t\right)
$$

we have the relation

$$
e_{q}\left(a D_{x}^{2}\right) e_{q}\left([2]_{q} x t\right)=e_{q}\left([2]_{q}^{2} a t^{2}\right) e_{q}\left([2]_{q} x t\right) .
$$

By choosing $a=-1 /[2]_{q}^{2}$ we get the result (26).

\section{Proposition 2.}

$$
H_{n}(x ; q)=[2]_{q}^{n} e_{q}\left(-\frac{1}{[2]_{q}^{2}} D_{x}^{2}\right) x^{n}
$$


Proof. The right-hand side of (26) is the generating function for the q-Hermite polynomials (10). Hence, equating the coefficients of $t^{n}$ on both sides gives the result.

\section{Proposition 3.}

$e_{q}\left(-\frac{D_{x}^{2}}{[2]_{q}^{2}}\right) x^{n+1}=\frac{1}{[2]_{q}}\left([2]_{q} x-\frac{\left(M_{q}+q^{\frac{n+1}{2}} M_{\sqrt{q}}\right) D_{x}}{[2]_{q}}\right) e_{q}\left(-\frac{D_{x}^{2}}{[2]_{q}^{2}}\right) x^{n}$

Proof. We use (30) and relation (20).

Corrollary 1. If the function $f(x)$ is expandable to the power series $f(x)=\sum_{n=0}^{\infty} a_{n} x^{n}$, then we have the next formal $q$-Hermite series representation

$$
e_{q}\left(-\frac{1}{[2]_{q}^{2}} D_{x}^{2}\right) f(x)=\sum_{n=0}^{\infty} a_{n} \frac{H_{n}(x ; q)}{[2]_{q}^{n}} .
$$

\section{4. q-Kampe de Feriet polynomials}

We define the q-Kampe de Feriet polynomials as

$$
H_{n}(x, v t ; q)=(-v t)^{\frac{n}{2}} H_{n}\left(\frac{x}{[2]_{q} \sqrt{-v t}} ; q\right),
$$

so that from (20) we obtain the next recursion formula

$$
H_{n+1}(x, v t ; q)=\left(x+\left(M_{q}+q^{\frac{n+1}{2}} M_{\sqrt{q}}\right) v t D_{x}\right) H_{n}(x, v t ; q) .
$$

By the recursion it gives

$$
H_{n}(x, v t ; q)=\prod_{k=1}^{n}\left(x+\left(M_{q}+q^{\frac{k}{2}} M_{\sqrt{q}}\right) v t D_{x}\right) \cdot 1
$$

or by notation (25)

$$
H_{n}(x, v t ; q)=\left(\left(x+M_{q} v t D_{x}\right)+q^{\frac{1}{2}} M_{\sqrt{q}} v t D_{x}\right)_{\sqrt{q}>}^{n} \cdot 1 .
$$

Then the first few polynomials are

$$
\begin{aligned}
& H_{0}(x, v t ; q)=1, \\
& H_{1}(x, v t ; q)=x, \\
& H_{2}(x, v t ; q)=x^{2}+[2]_{q} v t \\
& H_{3}(x, v t ; q)=x^{3}+[2]_{q}[3]_{q} v t x, \\
& H_{4}(x, v t ; q)=x^{4}+[3]_{q}[4]_{q} v t x^{2}+[2]_{q}[3]_{q}[2]_{q^{2}} v^{2} t^{2} .
\end{aligned}
$$

\section{5. q-heat equation}

We introduce the q-heat equation

$$
\left(D_{t}-v D_{x}^{2}\right) \phi(x, t)=0
$$

with partial $q$-derivatives with respect to $t$ and $x$. The solution of this equation expanded in terms of parameter $k$

$$
\phi(x, t)=e_{q}\left(v k^{2} t\right) e_{q}(k x)=\sum_{n=0}^{\infty} \frac{k^{n}}{[n] !} H_{n}(x, v t ; q)
$$


gives the set of q-Kampe de Feriet polynomial solutions for the equation. Then we find the time evolution of zeros $x_{k}(t)$ for these polynomials in terms of zeros $z_{k}(n, q)$ of the q-Hermite polynomials:

$$
H_{n}\left(z_{k}(n, q), q\right)=0
$$

so that

$$
x_{k}(t)=[2] z_{k}(n, q) \sqrt{-v t} .
$$

For $n=2$ we have two zeros determined by q-numbers,

$$
\begin{aligned}
& x_{1}(t)=\sqrt{[2]_{q}} \sqrt{-v t}, \\
& x_{2}(t)=-\sqrt{[2]_{q}} \sqrt{-v t},
\end{aligned}
$$

and moving in opposite directions according to (38). For $n=3$ we have zeros determined by q-numbers:

$$
\begin{aligned}
& x_{1}(t)=-\sqrt{[3]_{q} !} \sqrt{-v t} \\
& x_{2}(t)=0 \\
& x_{3}(t)=\sqrt{[3]_{q} !} \sqrt{-v t}
\end{aligned}
$$

two of which are moving in the opposite direction according to (38) and one is at rest.

\section{Evolution operator}

Following similar calculations as in proposition 1 we have the next relation

$$
e_{q}\left(v t D_{x}^{2}\right) e_{q}(k x)=e_{q}\left(v t k^{2}\right) e_{q}(k x) .
$$

The right-hand side of this expression is the plane-wave-type solution (36) of the q-heat equation (35). Equating the coefficients of $k^{n}$ on both sides we get the q-Kampe de Feriet polynomial solutions of equation

$$
H_{n}(x, v t ; q)=e_{q}\left(v t D_{x}^{2}\right) x^{n}
$$

Consider an arbitrary, expandable to the power series function $f(x)=\sum_{n=0}^{\infty} a_{n} x^{n}$; then, the formal series

$$
\begin{aligned}
f(x, t)=e_{q}\left(v t D_{x}^{2}\right) f(x) & =\sum_{n=0}^{\infty} a_{n} e_{q}\left(v t D_{x}^{2}\right) x^{n} \\
& =\sum_{n=0}^{\infty} a_{n} H_{n}(x, v t ; q)
\end{aligned}
$$

represents a time-dependent solution of the q-heat equation (35). The domain of convergency for this series is determined by asymptotic properties of our q-Kampe de Feriet polynomials for $n \rightarrow \infty$ and requires additional study.

According to this we have the evolution operator for the q-heat equation as

$$
U(t)=e_{q}\left(v t D_{x}^{2}\right)
$$

It allows us to solve the initial value problem 


$$
\begin{aligned}
& \left(D_{t}-v D_{x}^{2}\right) \phi(x, t)=0, \\
& \phi\left(x, 0^{+}\right)=f(x),
\end{aligned}
$$

in the form

$$
\phi(x, t)=e_{q}\left(v t D_{x}^{2}\right) \phi\left(x, 0^{+}\right)=e_{q}\left(v t D_{x}^{2}\right) f(x),
$$

where we imply the base $q>1$ so that $e_{q}(x)$ is an entire function.

\section{7. q-Burgers' type equation}

We introduce the q-Cole-Hopf transformation

$$
u(x, t)=-2 v \frac{D_{x} \phi(x, t)}{\phi(x, t)},
$$

where $\phi(x, t)$ is the solution of the q-heat equation (35).

Then $u(x, t)$ satisfies the q-Burgers' type equation with cubic nonlinearity

$$
\begin{aligned}
D_{t} u(x, t)-v & D_{x}^{2} u(x, t)=\frac{1}{2}\left[\left(u(x, q t)-u(x, t) M_{q}^{x}\right) D_{x} u(x, t)\right] \\
& -\frac{1}{2}\left[D_{x}(u(q x, t) u(x, t))\right]+\frac{1}{4 v}\left[u\left(q^{2} x, t\right)-u(x, q t)\right] u(q x, t) u(x, t) .
\end{aligned}
$$

When $q \rightarrow 1$ it reduces to the standard Burgers' equation

$$
u_{t}+u u_{x}=v u_{x x} .
$$

\subsection{IVP for q-Burgers' type equation}

Substituting the operator solution (51) to (52) we find the operator solution for the q-Burgerstype equation in the form

$$
u(x, t)=-2 v \frac{e_{q}\left(v t D_{x}^{2}\right) D_{x} f(x)}{e_{q}\left(v t D_{x}^{2}\right) f(x)} .
$$

This solution corresponds to the initial function

$$
u\left(x, 0^{+}\right)=-2 v \frac{D_{x} f(x)}{f(x)} .
$$

Thus, for arbitrary initial value $u\left(x, 0^{+}\right)=F(x)$ for the q-Burgers equation we need to solve the initial value problem for the q-heat equation (35) with the initial function $f(x)$ satisfying the first-order q-difference equation

$$
\left(D_{x}+\frac{1}{2 v} F(x)\right) f(x)=0 .
$$

\section{8. q-shock soliton solution}

As a particular solution of the q-heat equation we choose first

$$
\phi(x, t)=e_{q}\left(k^{2} t\right) e_{q}(k x) ;
$$

then, we find the solution of the q-Burgers equation as a constant

$$
u(x, t)=-2 v k .
$$




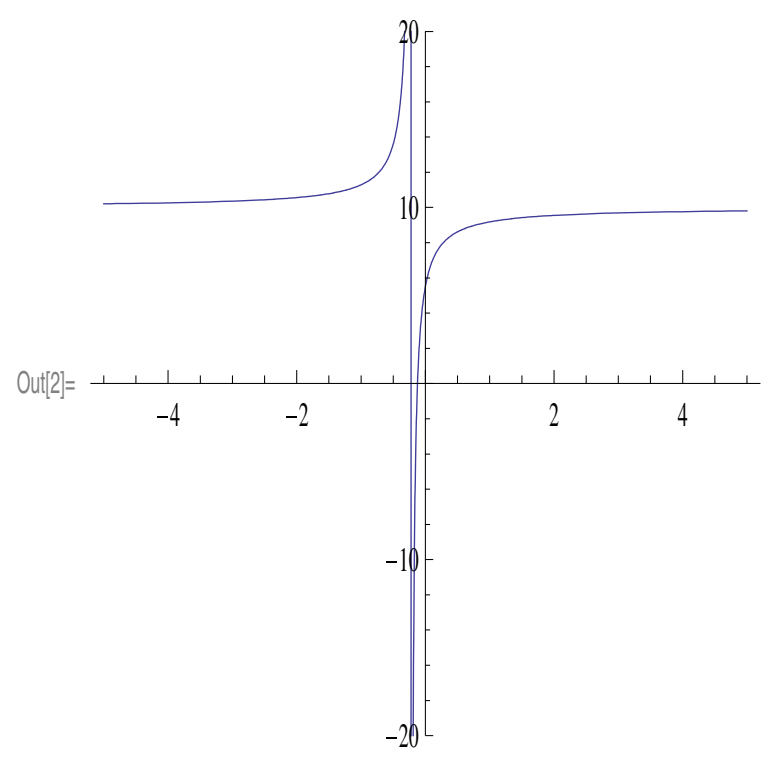

Figure 1. Singular q-shock soliton.

We note that for this solution of the q-heat equation, we have an infinite set of zeros, and the space position of zeros is fixed during time evolution at points $x_{n}=-q^{n+1} /(q-1) k$, $n=0,1, \ldots$

If we choose the linear superposition

$$
\phi(x, t)=e_{q}\left(k_{1}^{2} t\right) e_{q}\left(k_{1} x\right)+e_{q}\left(k_{2}^{2} t\right) e_{q}\left(k_{2} x\right),
$$

then we have the q-shock soliton solution

$$
u(x, t)=-2 v \frac{k_{1} e_{q}\left(k_{1}^{2} t\right) e_{q}\left(k_{1} x\right)+k_{2} e_{q}\left(k_{2}^{2} t\right) e_{q}\left(k_{2} x\right)}{e_{q}\left(k_{1}^{2} t\right) e_{q}\left(k_{1} x\right)+e_{q}\left(k_{2}^{2} t\right) e_{q}\left(k_{2} x\right)} .
$$

This expression is the q-analog of the Burgers shock soliton and for $q \rightarrow 1$ it reduces to the last one. However, in contrast to the standard Burgers case, due to zeros of the q-exponential function this expression admits singularities for some values of parameters $k_{1}$ and $k_{2}$. In figure 1 we plot the singular q-shock soliton for $k_{1}=1$ and $k_{2}=10$ at time $t=0$ with base $q=10$.

It turns out that for some specific values of parameters we can find the regular q-shock soliton solution. We introduce the cosine q-hyperbolic function

$$
\cosh _{q}(x)=\frac{e_{q}(x)+e_{q}(-x)}{2}
$$

or

$$
\cosh _{q}(x)=\frac{1}{2}\left(e_{q}(x)+\frac{1}{e_{\frac{1}{q}}(x)}\right),
$$

and then by using the infinite product representation (4) for the q-exponential function we have

$$
\cosh _{q}(x)=\frac{1}{2}\left(\left(1+\left(1-\frac{1}{q}\right) x\right)_{1 / q}^{\infty}+\left(1-\left(1-\frac{1}{q}\right) x\right)_{q}^{\infty}\right)
$$




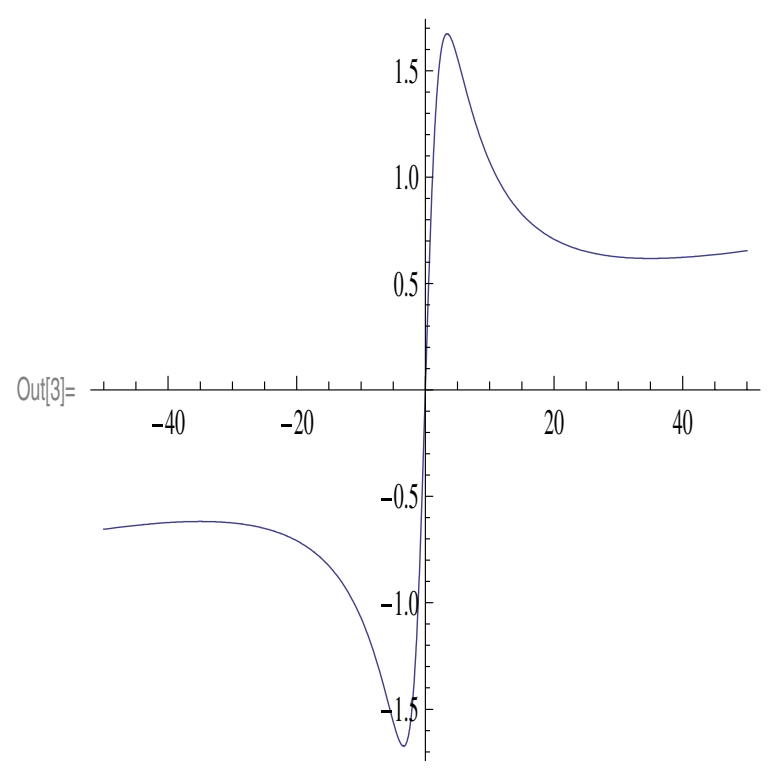

Figure 2. The regular q-shock soliton for $k_{1}=1, k_{2}=-1$, at range $(-50,50)$.

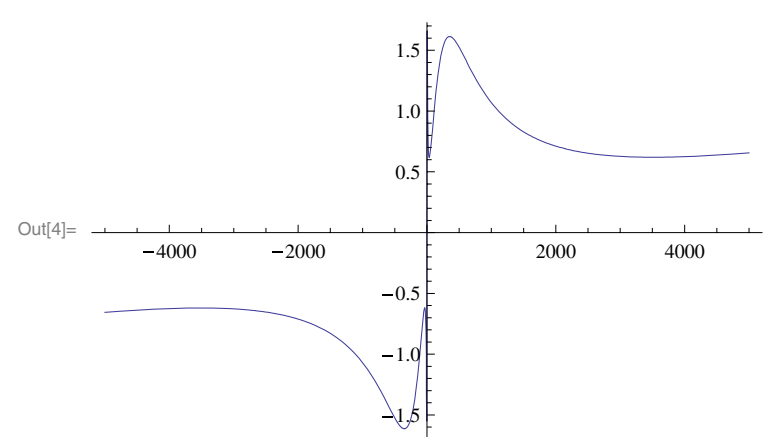

Figure 3. The regular q-shock soliton for $k_{1}=1, k_{2}=-1$ at range $(-5000,5000)$.

From (5),(6) we find that zeros of the first product are located on the negative axis $x$, while for the second product on the positive axis $x$. Therefore, the function has no zeros for real $x$ and $\cosh _{q}(0)=1$.

If we choose $k_{1}=1$ and $k_{2}=-1$, the time-dependent factors in the numerator and the denominator of (60) cancel each other and we have the stationary shock soliton

$$
u(x, t)=-2 v \frac{e_{q}(x)-e_{q}(-x)}{e_{q}(x)+e_{q}(-x)} \equiv-2 v \tanh _{q}(x) .
$$

Due to the above consideration this function has no singularity on the real axis and everywhere we have regular q-shock soliton solution.

In figures 2-4 we plot the regular q-shock soliton for $k_{1}=1$ and $k_{2}=-1$ at different ranges of $x$ and $q=10$. It is a remarkable fact that the structure of our shock soliton shows self-similarity property in the space coordinate $x$. Indeed at the ranges of parameter $x=50,5000,500000$ the structure of shock looks almost the same. 


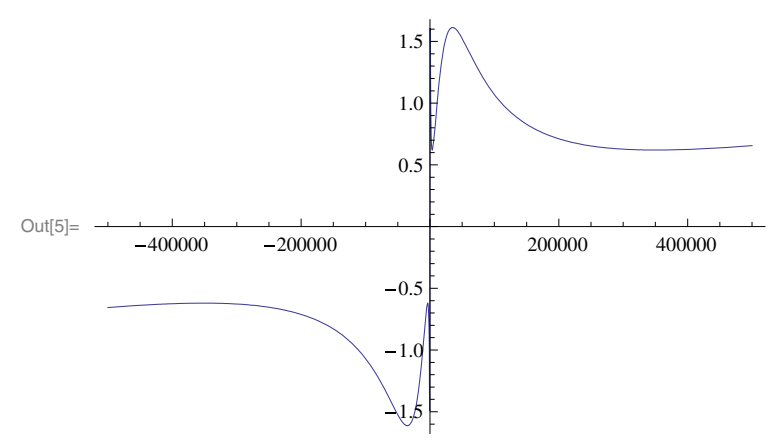

Figure 4. The regular q-shock soliton for $k_{1}=1, k_{2}=-1$ at range $(-500000,500000)$

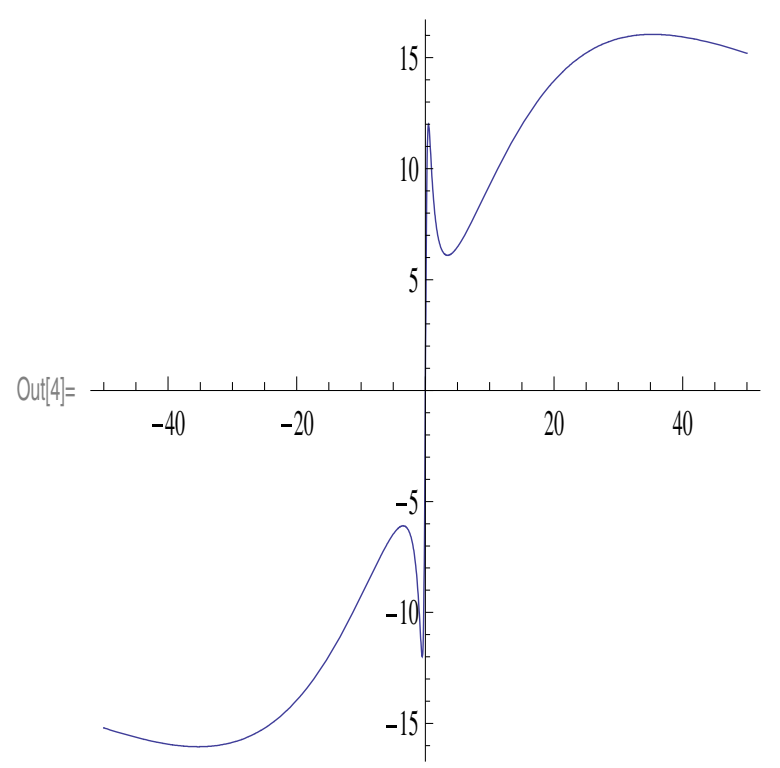

Figure 5. Multi q-shock regular for $k_{1}=1, k_{2}=-1, k_{3}=10, k_{4}=-10$ at $t=0$.

For the set of arbitrary numbers $k_{1}, \ldots, k_{N}$

$$
\phi(x, t)=\sum_{n=1}^{N} e_{q}\left(k_{n}^{2} t\right) e_{q}\left(k_{n} x\right),
$$

we have a multi-shock solution in the form

$$
u(x, t)=-2 v \frac{\sum_{n=1}^{N} k_{n} e_{q}\left(k_{n}^{2} t\right) e_{q}\left(k_{n} x\right)}{\sum_{n=1}^{N} e_{q}\left(k_{n}^{2} t\right) e_{q}\left(k_{n} x\right)} .
$$

In general this solution admits several singularities. To have a regular multi-shock solution we can consider the even number of terms $N=2 k$ with opposite wave numbers. When $N=$ 4 and $k_{1}=1, k_{2}=-1, k_{3}=10, k_{4}=-10$ we have the q-multi-shock soliton solution

$$
u(x, t)=-2 v \frac{e_{q}(t) \sinh _{q}(x)+10 e_{q}(100 t) \sinh _{q}(10 x)}{e_{q}(t) \cosh _{q}(x)+e_{q}(100 t) \cosh _{q}(10 x)} .
$$

In figure 5 we plot the $N=4$ case with values of the wave numbers $k_{1}=1, k_{2}=-1$, $k_{3}=10, k_{4}=-10$ at $t=0$ and $q=10$. To have a regular solution for any time $t$ and given 
base $q$, we should choose proper numbers $k_{i}$ which are not in the form of the power of $q$. This question is under study now.

\section{Acknowledgments}

One of the authors (SN) was partially supported by National Scholarship of the Scientific and Technological Research Council of Turkey (TUBITAK). This work was supported partially by Izmir Institute of Technology, Turkey. The authors would like to thank the referees for constructive comments.

\section{References}

[1] Kac V and Cheung P 2002 Quantum Calculus (New York: Springer)

[2] Exton H 1983 q-Hypergeometric Functions and Applications (New York: Wiley)

[3] Jackson F H 1904 A basic sine and cosine with symbolic solutions of certain differential equations Proc. Edinburgh Math. Soc. 22 28-39

[4] Rajkovic P and Marinkovic S 2001 On q-analogies of generalized Hermite's polynomials Filomat 15277

[5] Cigler J and Zeng J 2009 Two curious q-analogues of Hermite polynomials, arXiv:0905.0228

[6] Negro J 1996 The factorization method and hierarchies of $q$-oscillator Hamiltonians Centre de Recherches Mathematiques CRM Proceedings and Lecture Notes vol 9 p 239

[7] Ismail M 2005 Classical and Quantum Orthogonal Polynomials in One Variable (Cambridge: Cambridge University Press) 